Supporting Information

\title{
Possible Peroxo State of the Dicopper Site of Particulate Methane Monooxygenase from Combined Quantum Mechanics and Molecular Mechanics Calculations
}

Shuhei Itoyama, ${ }^{1}$ Kazuki Doitomi, ${ }^{1}$ Takashi Kamachi, ${ }^{1}$ Yoshihito Shiota, ${ }^{1}$ Kazunari Yoshizawa*,1,2

${ }^{1}$ Institute for Materials Chemistry and Engineering and International Research Center for Molecular Systems, Kyushu University, Nishi-ku, Fukuoka 819-0395, Japan

${ }^{2}$ Elements Strategy Initiative for Catalysts and Batteries (ESICB), Kyoto University, Nishikyo-ku, Kyoto 615-8520, Japan

*Author to whom correspondence should be addressed.

kazunari@ms.ifoc.kyushu-u.ac.jp (K.Y.)

The supporting information includes:

The detailed information of the TD-DFT calculation

The QM region of QM/MM optimized structures 
The detailed information of the TD-DFT calculation

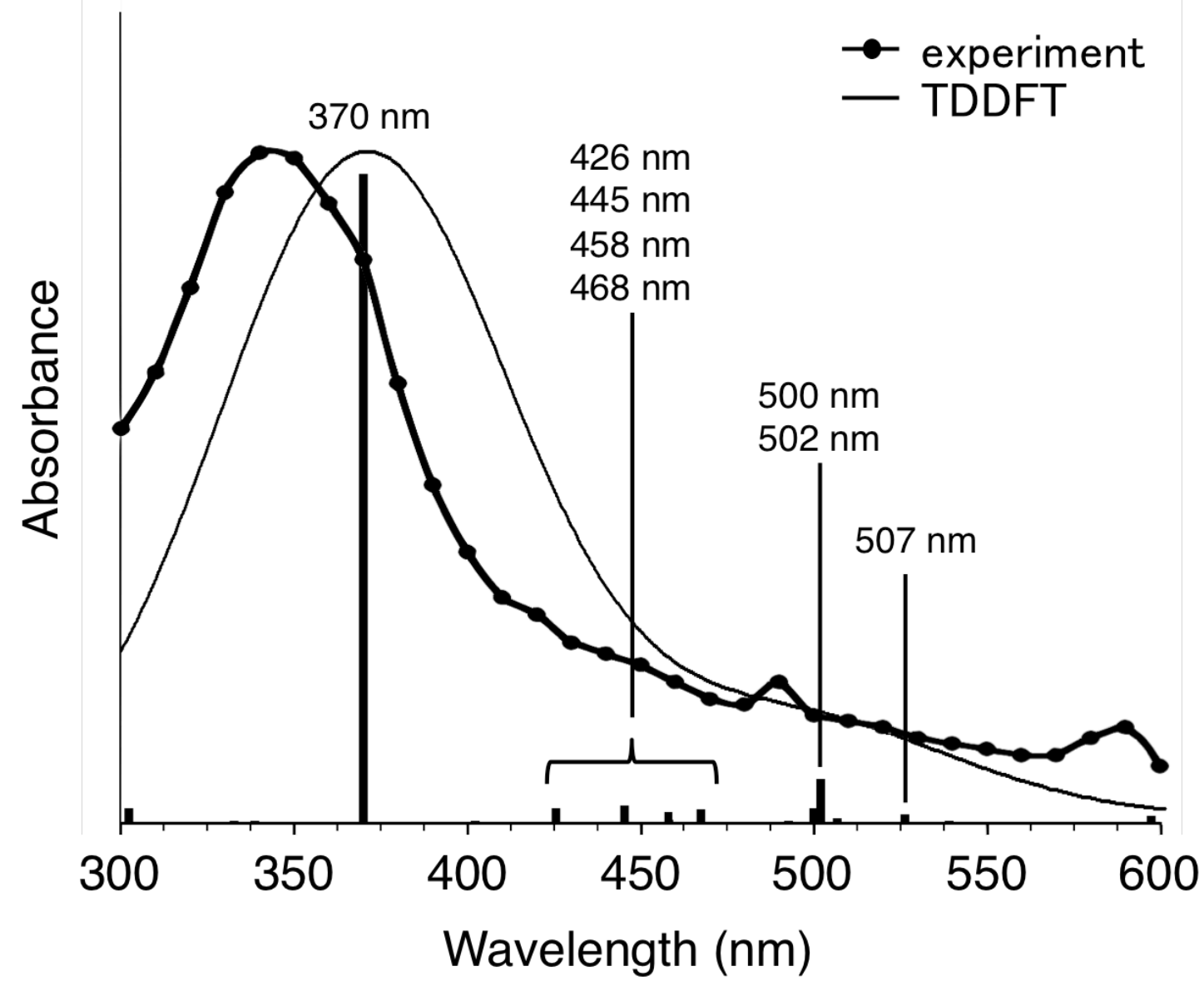

Figure S1. Observed optical spectrum for $\mathrm{O}_{2}$ adduct of pMMO and TD-DFT analysis for a $\mathrm{QM} / \mathrm{MM}$ optimized structure of the $\mu-\eta^{2}: \eta^{2}-$ peroxo- $\mathrm{Cu}_{2}{ }_{2}$ state of $\mathrm{pMMO}$. The $y$-axis is normalized to the main absorption peak. The vertical lines in the $y$-axis indicate calculated oscillator strengths.. 
Details of main excitations:

$370.28 \mathrm{~nm} \quad \mathrm{f}=0.44500$

Dominant contributions:

$\begin{array}{lllll}\text { occ. orbital } & \mathrm{eV} & \text { virt. orbital } & \mathrm{eV} & \text { |coeff.|^2*100 } \\ \beta \text {-HOMO-9 } & -6.61 & \beta \text {-LUMO } & -2.62 & 47.3 \\ \alpha \text {-HOMO-9 } & -6.46 & \alpha \text {-LUMO } & -2.76 & 14.8\end{array}$

$425.75 \mathrm{~nm} \quad \mathrm{f}=0.01069$

Dominant contributions:

$\begin{array}{lllll}\text { occ. orbital } & \mathrm{eV} & \text { virt. orbital } & \mathrm{eV} & \text { Icoeff.|^ } 2 * 100 \\ \beta \text {-HOMO-6 } & -6.25 & \beta \text {-LUMO } & -2.62 & 57.8 \\ \beta \text {-HOMO-28 } & -8.98 & \beta \text {-LUMO } & -2.62 & 10.5 \\ \beta \text {-HOMO-19 } & -8.45 & \beta \text {-LUMO } & -2.62 & 10.0\end{array}$

$445.40 \mathrm{~nm} \quad \mathrm{f}=0.01245$

Dominant contributions:

$\begin{array}{lllll}\text { occ. orbital } & \mathrm{eV} & \text { virt. orbital } & \mathrm{eV} & \text { Icoeff.|^ } 2 * 100 \\ \beta \text {-HOMO-6 } & -6.25 & \beta \text {-LUMO } & -2.62 & 29.5 \\ \beta \text {-HOMO-19 } & -8.45 & \beta \text {-LUMO } & -2.62 & 16.0 \\ \beta \text {-HOMO-28 } & -8.98 & \beta \text {-LUMO } & -2.62 & 10.5\end{array}$

$458.13 \mathrm{~nm} \quad \mathrm{f}=0.00782$

Dominant contributions:

$\begin{array}{lllll}\text { occ. orbital } & \mathrm{eV} & \text { virt. orbital } & \mathrm{eV} & \text { Icoeff.|^ } 2 * 100 \\ \alpha \text {-HOMO-5 } & -6.02 & \alpha \text {-LUMO } & -2.76 & 28.3 \\ \alpha \text {-HOMO-6 } & -6.24 & \alpha \text {-LUMO } & -2.76 & 17.0 \\ \alpha \text {-HOMO-9 } & -6.46 & \alpha \text {-LUMO } & -2.76 & 11.7\end{array}$

$467.81 \mathrm{~nm} \quad \mathrm{f}=0.00937$

Dominant contributions:

$\begin{array}{lllll}\text { occ. orbital } & \mathrm{eV} & \text { virt. orbital } & \mathrm{eV} & \text { Icoeff.|^ } 2 * 100 \\ \alpha-\mathrm{HOMO}-4 & -5.97 & \alpha \text {-LUMO } & -2.76 & 39.3 \\ \alpha-\mathrm{HOMO}-5 & -6.02 & \alpha \text {-LUMO } & -2.76 & 31.5\end{array}$

$500.14 \mathrm{~nm} \quad \mathrm{f}=0.01024$

Dominant contributions: 


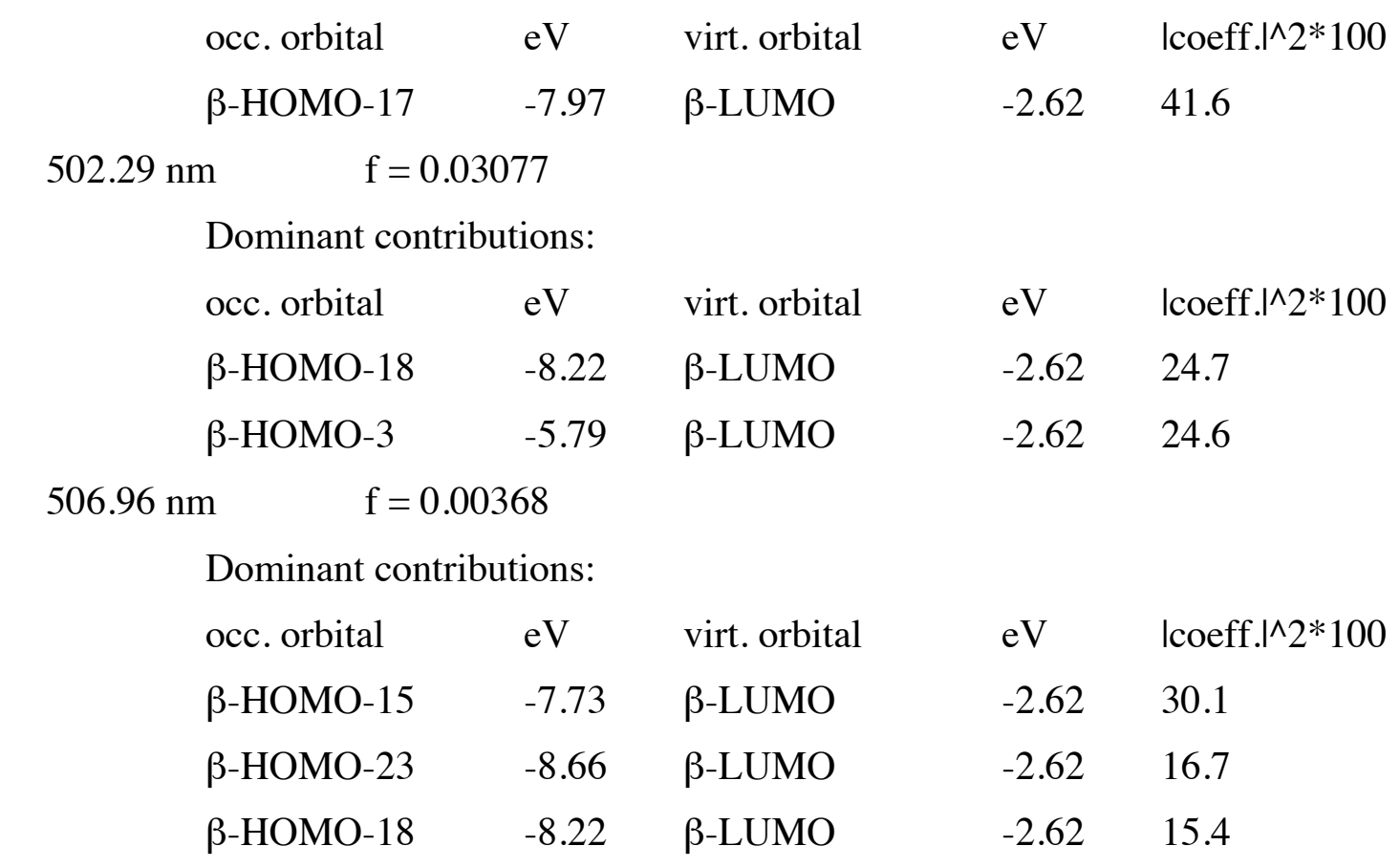


The QM region of QM/MM-optimized structures

Units in $\AA$

The resting state of $\mathrm{Cu}_{2}^{\mathrm{I}}$ at the B3LYP-D/TZVP level of theory $\mathrm{E}=-4790.310934$ (a.u.)

$\mathrm{N} \quad 37.742434130 .61220581 .401311$

C $\quad 39.032212131 .35506381 .530639$

H $\quad 38.819473132 .31661581 .062659$

C $\quad 39.404600131 .62440282 .988896$

$\mathrm{H} \quad 40.450517131 .91597783 .071907$

$\mathrm{H} \quad 39.298551130 .71381783 .589519$

C $\quad 38.577111132 .72472383 .593595$

$\mathrm{N} \quad 37.183437132 .69367083 .646869$

C $\quad 36.789151133 .79478984 .266873$

H $\quad 35.769270134 .06172484 .488359$

$\mathrm{N} \quad 37.859623134 .54797984 .592450$

H $\quad 37.834551135 .42496685 .091488$

C $\quad 38.998215133 .88884984 .172076$

$\mathrm{H} \quad 39.984493134 .29188784 .311078$

$\mathrm{H} \quad 37.528614130 .63061680 .395377$

$\mathrm{H} \quad 37.885045129 .64569781 .682117$

C $\quad 38.095450133 .63009676 .663494$

$\mathrm{H} \quad 38.259462132 .61890176 .289898$

$\mathrm{H} \quad 37.804541134 .23155075 .796740$

C $\quad 36.943629133 .62338377 .680009$

$\mathrm{H} \quad 36.081579133 .16577977 .191010$

$\mathrm{H} \quad 36.613230134 .61780377 .985313$

C $\quad 37.246445132 .81138778 .936434$

O $\quad 37.527454131 .61062678 .797995$

O $\quad 37.202305133 .44159880 .061197$

C $\quad 34.395682130 .05274486 .590789$

H $\quad 35.253834130 .23966085 .936896$ 

H $\quad 34.768696130 .16711387 .613363$
C $\quad 33.394619131 .16552986 .373441$
$\mathrm{N} \quad 33.291058131 .85943585 .170341$
C $\quad 32.507200132 .91938485 .385612$
$\mathrm{H} \quad 32.224852133 .67292484 .670258$
$\mathrm{N} \quad 32.104476132 .95158286 .655681$
$\mathrm{H} \quad 31.574181133 .74691087 .044792$
C $\quad 32.651822131 .85934187 .295585$
H $\quad 32.511428131 .68525188 .344513$
C $\quad 33.072608128 .29395379 .325511$
$\mathrm{H} \quad 32.605543128 .63887078 .403336$
$\mathrm{H} \quad 33.976316127 .74342979 .052642$
C $\quad 33.455002129 .46437780 .166678$
C $\quad 34.341872129 .60635881 .188305$
H $\quad 34.992253128 .87448881 .632080$
N $\quad 32.937693130 .73009379 .957437$
$\mathrm{H} \quad 32.259552130 .95228879 .235708$
C $\quad 33.500392131 .59537380 .809721$
H $\quad 33.374555132 .66769180 .785544$
$\mathrm{N} \quad 34.373014130 .94209081 .597399$
C $\quad 33.277435139 .65460581 .362070$
H $\quad 32.756729140 .17494180 .559564$
$\mathrm{H} \quad 32.631979139 .69311982 .244047$
C $\quad 33.563702138 .20639581 .008756$
C $\quad 33.365672137 .70172279 .721317$
$\mathrm{H} \quad 32.938333138 .33878378 .955969$
C $\quad 33.692537136 .38455879 .397120$
$\mathrm{H} \quad 33.527692136 .00124078 .397079$
C $\quad 34.045712137 .32049081 .983076$
H $\quad 34.157247137 .66744083 .004299$
C $\quad 34.372939136 .00653981 .672736$
$\mathrm{H} \quad 34.726210135 .32412682 .439200$
C $\quad 34.219937135 .54283080 .367756$ 

O 34.531189134 .22486180 .042386
H $\quad 35.488152134 .04658780 .177213$
$\mathrm{Cu} \quad 36.172878131 .64682782 .318987$
$\mathrm{Cu} \quad 33.962743131 .41297483 .467812$

The resting state of $\mathrm{Cu}_{2}^{\mathrm{I}}$ at the BP86-D/TZVP level of theory $\mathrm{E}=-4792.003138$ (a.u.)
$\mathrm{N} \quad 37.708088130 .65014781 .380264$
C $\quad 39.021073131 .36079981 .522245$
H $\quad 38.819863132 .34344481 .068073$
C $\quad 39.394774131 .59891182 .988067$
$\mathrm{H} \quad 40.448857131 .88483683 .084146$
H $\quad 39.277511130 .67016383 .572598$
C $\quad 38.563118132 .69051983 .602725$
N $\quad 37.164793132 .64442583 .650937$
C $\quad 36.748461133 .74941084 .273306$
$\mathrm{H} \quad 35.716048133 .99708184 .494240$
N $\quad 37.818419134 .51567884 .603475$
H $\quad 37.783563135 .39844285 .107396$
C $\quad 38.970650133 .86794984 .186704$
H 39.957951134 .28803184 .328246
H $\quad 37.491828130 .71867080 .360068$
H $\quad 37.838621129 .66183881 .617775$
C $\quad 38.081046133 .63157376 .682001$
H $\quad 38.237158132 .60721776 .317968$
H $\quad 37.782875134 .22403075 .802263$
C $\quad 36.935328133 .64100077 .709179$
H $\quad 36.054679133 .19802177 .223161$
$\mathrm{H} \quad 36.610108134 .64151978 .028180$
C $\quad 37.228812132 .80861278 .957512$
O $\quad 37.519208131 .60046478 .798183$
O $\quad 37.164765133 .41917380 .104747$
C $\quad 34.393352130 .05173286 .556843$ 


$$
\begin{aligned}
& \mathrm{H} \quad 35.248891130 .23749385 .885681 \\
& \text { H } \quad 34.779982130 .18352387 .580556 \\
& \text { C } \quad 33.390126131 .16374386 .335339 \\
& \mathrm{~N} \quad 33.307361131 .86947485 .130106 \\
& \text { C } \quad 32.523106132 .94298485 .339615 \\
& \text { H } \quad 32.260678133 .71170184 .622196 \\
& \mathrm{~N} \quad 32.099960132 .96622686 .611846 \\
& \text { H } \quad 31.568073133 .77023087 .001604 \\
& \text { C } \quad 32.632127131 .86347187 .255357 \\
& \mathrm{H} \quad 32.469922131 .68352088 .308195 \\
& \text { C } \quad 33.065303128 .30299379 .336963 \\
& \text { H } \quad 32.591352128 .65699378 .413474 \\
& \text { H } \quad 33.971156127 .74840879 .050051 \\
& \text { C } \quad 33.464862129 .46841880 .179224 \\
& \text { C } \quad 34.370015129 .59552281 .199837 \\
& \text { H } \quad 35.017750128 .84983981 .641643 \\
& \text { N } \quad 32.962405130 .74511779 .975433 \\
& \mathrm{H} \quad 32.277710130 .97788079 .253457 \\
& \text { C } \quad 33.543231131 .61210680 .829206 \\
& \mathrm{H} \quad 33.459484132 .69766380 .781218 \\
& \mathrm{~N} \quad 34.428365130 .93687081 .609828 \\
& \text { C } \quad 33.283960139 .64219781 .366520 \\
& \mathrm{H} \quad 32.745196140 .17326080 .573176 \\
& \text { H } \quad 32.641873139 .66559782 .261292 \\
& \text { C } \quad 33.566727138 .19717680 .987992 \\
& \text { C } \quad 33.316091137 .70176079 .697865 \\
& \mathrm{H} \quad 32.850140138 .34884078 .951923 \\
& \text { C } \quad 33.638768136 .38529979 .342066 \\
& \text { H } \quad 33.438092136 .01449778 .335482 \\
& \text { C } \quad 34.091631137 .29510181 .935606 \\
& \text { H } \quad 34.242626137 .63091882 .963788 \\
& \text { C } \quad 34.410294135 .97833381 .596667 \\
& \text { H } \quad 34.797371135 .28024582 .345324
\end{aligned}
$$


C $\quad 34.213567135 .52921680 .284052$

O $\quad 34.527218134 .21726479 .918417$

H $\quad 35.477019134 .01764980 .147585$

$\mathrm{Cu} \quad 36.166795131 .61853482 .349944$

$\mathrm{Cu} \quad 34.008084131 .43061783 .454517$

The resting state of $\mathrm{Cu}_{2}^{\mathrm{I}}$ at the B97D/TZVP level of theory

$\mathrm{E}=-4791.788204$ (a.u.)

$\mathrm{N} \quad 37.748254130 .62224481 .380041$

C $\quad 39.056198131 .34528581 .521085$

H $\quad 38.850258132 .32297081 .070842$

C $\quad 39.432166131 .58029382 .989721$

H $\quad 40.483741131 .86618283 .080629$

H $\quad 39.313907130 .65307083 .570635$

C $\quad 38.602890132 .67544483 .605967$

$\mathrm{N} \quad 37.205556132 .62486383 .680835$

C $\quad 36.800050133 .74167184 .284978$

H $\quad 35.776475133 .99668584 .522236$

$\mathrm{N} \quad 37.871316134 .51983484 .575824$

H $\quad 37.843727135 .40722485 .062134$

C $\quad 39.017704133 .86548184 .152946$

$\mathrm{H} \quad 40.002163134 .29034984 .272014$

H $\quad 37.526415130 .68158680 .369638$

$\mathrm{H} \quad 37.885547129 .64234081 .624341$

C $\quad 38.133821133 .63012876 .642004$

$\mathrm{H} \quad 38.300133132 .61435776 .268191$

$\mathrm{H} \quad 37.844122134 .23293875 .770063$

C $\quad 36.968772133 .61764377 .652564$

$\mathrm{H} \quad 36.112549133 .15383377 .150504$

H $\quad 36.623822134 .61294077 .956593$

C $\quad 37.267614132 .79932278 .916099$

O $\quad 37.536427131 .58735678 .770099$

O $\quad 37.230833133 .43286380 .045516$ 

C $\quad 34.381914130 .03832286 .604977$
H $\quad 35.239941130 .23468785 .945025$
H $\quad 34.757836130 .15453287 .631562$
C $\quad 33.374017131 .15171586 .390288$
$\mathrm{N} \quad 33.289729131 .85767785 .186718$
C $\quad 32.507629132 .92952085 .397776$
H $\quad 32.240187133 .69397984 .682079$
$\mathrm{N} \quad 32.087035132 .95370686 .669526$
$\mathrm{H} \quad 31.558156133 .75356287 .057566$
C $\quad 32.619962131 .84970987 .312380$
$\mathrm{H} \quad 32.464317131 .67536188 .363648$
C $\quad 33.060521128 .27899179 .327439$
$\mathrm{H} \quad 32.592637128 .63247478 .404213$
H $\quad 33.965754127 .72342179 .050400$
C $\quad 33.454027129 .44502980 .175635$
C $\quad 34.350606129 .57107881 .201236$
H $\quad 35.001865128 .82916181 .637863$
$\mathrm{N} \quad 32.943498130 .71991979 .977647$
$\mathrm{H} \quad 32.263252130 .95270379 .257926$
C $\quad 33.514925131 .58049480 .841784$
H $\quad 33.394704132 .65692280 .822042$
N $\quad 34.393635130 .90822481 .624757$
C $\quad 33.264895139 .66850981 .359799$
H $\quad 32.731801140 .20950780 .573410$
H $\quad 32.629764139 .68609582 .255981$
C $\quad 33.538436138 .22363080 .966387$
C $\quad 33.308302137 .74908079 .665458$
H $\quad 32.868239138 .41004278 .921531$
C $\quad 33.626591136 .43449079 .298439$
H $\quad 33.441963136 .07798878 .286825$
C $\quad 34.038032137 .30751581 .912510$
H $\quad 34.170529137 .62962582 .944180$
C $\quad 34.356253135 .99375281 .561475$ 
$\mathrm{H} \quad 34.723607135 .28820082 .308228$

C $\quad 34.177101135 .56323680 .240291$

O $\quad 34.485605134 .25141679 .862286$

$\mathrm{H} \quad 35.420555134 .05211280 .092523$

$\mathrm{Cu} \quad 36.188657131 .58392882 .359643$

$\mathrm{Cu} \quad 33.987676131 .39698783 .498283$

The resting state of $\mathrm{Cu}_{2}^{\mathrm{I}}$ at the M06-D/TZVP level of theory

$\mathrm{E}=-4790.141831$ (a.u.)

$\mathrm{N} \quad 37.843194130 .44948581 .533250$

C $\quad 39.094696131 .23394581 .501789$

$\mathrm{H} \quad 38.822356132 .16833380 .997359$

C $\quad 39.549620131 .60401182 .898767$

H $\quad 40.590929131 .92747882 .899701$

H $\quad 39.518939130 .72954983 .560525$

C $\quad 38.733473132 .71322683 .484296$

$\mathrm{N} \quad 37.347850132 .68885083 .573481$

C $\quad 36.984793133 .80142484 .175470$

H $\quad 35.971715134 .08733384 .416342$

$\mathrm{N} \quad 38.061767134 .55951484 .454957$

H $\quad 38.058719135 .44589484 .936238$

C $\quad 39.177198133 .88396984 .021303$

$\mathrm{H} \quad 40.171970134 .27534884 .133803$

$\mathrm{H} \quad 37.572605130 .27887280 .564760$

H $\quad 38.002847129 .56691682 .007379$

C $\quad 37.709363133 .63553876 .985499$

H $\quad 37.792409132 .60147076 .653599$

$\mathrm{H} \quad 37.343708134 .19777076 .120694$

C $\quad 36.713287133 .72618778 .129964$

H $\quad 35.732217133 .42452277 .747535$

H $\quad 36.553850134 .73710578 .517096$

C $\quad 37.008798132 .79673779 .292584$

O $\quad 37.311960131 .63154479 .050215$ 
O $\quad 36.910101133 .30710680 .476953$

C $\quad 34.482342130 .03216486 .454666$

H $\quad 35.344159130 .18602785 .795735$

H $\quad 34.864362130 .16462387 .472685$

C $\quad 33.503550131 .14283386 .221302$

$\mathrm{N} \quad 33.452680131 .86198985 .036033$

C $\quad 32.663286132 .91295885 .246788$

$\mathrm{H} \quad 32.413496133 .69254684 .542338$

N $\quad 32.206777132 .91336686 .493605$

$\mathrm{H} \quad 31.658268133 .69634686 .876834$

C $\quad 32.721836131 .80921487 .123644$

H $\quad 32.532265131 .60114988 .161069$

C $\quad 33.238598128 .24165679 .289008$

H $\quad 32.805917128 .56560678 .340900$

H $\quad 34.134182127 .66006079 .064849$

C $\quad 33.608871129 .42242680 .100845$

C $\quad 34.528944129 .62735881 .078263$

$\mathrm{H} \quad 35.236537128 .93549781 .504671$

N $\quad 32.996578130 .64086379 .915439$

$\mathrm{H} \quad 32.269323130 .80865079 .228617$

C $\quad 33.535636131 .53857780 .741275$

H $\quad 33.304527132 .59673680 .744160$

$\mathrm{N} \quad 34.485690130 .95847081 .482421$

C $\quad 33.234712139 .70969081 .361445$

H 32.729329140 .23654680 .554028

$\mathrm{H} \quad 32.589528139 .77821082 .241998$

C $\quad 33.476129138 .26228381 .034088$

C $\quad 33.222567137 .73015279 .774050$

$\mathrm{H} \quad 32.786256138 .36009679 .006220$

C $\quad 33.504214136 .40250679 .479544$

$\mathrm{H} \quad 33.296670135 .99487078 .495975$

C $\quad 33.970195137 .39827582 .014036$

H $\quad 34.121631137 .77888483 .019656$ 
C $\quad 34.258595136 .07603381 .733608$

H $\quad 34.619647135 .39948082 .506449$

C $\quad 34.039693135 .58155580 .455611$

O $\quad 34.296913134 .25864080 .165876$

$\mathrm{H} \quad 35.196402134 .02488780 .471520$

$\mathrm{Cu} \quad 36.299368131 .78762082 .128532$

$\mathrm{Cu} \quad 34.154530131 .43272783 .348373$

The resting state of $\mathrm{Cu}^{\mathrm{I}} \mathrm{Cu}^{\mathrm{II}}$ at the B3LYP-D/TZVP level of theory

$\mathrm{E}=-4790.111037$ (a.u.)

N $\quad 37.669920130 .70175882 .049396$

C $\quad 38.932755131 .48929981 .897402$

H $\quad 38.651052132 .35176181 .290636$

C $\quad 39.448255131 .99749683 .236783$

H $\quad 40.410167132 .48985583 .092383$

H $\quad 39.655201131 .16666583 .920010$

C $\quad 38.530008132 .98251183 .924605$

N $\quad 37.140735132 .98545783 .809029$

C $\quad 36.666755133 .92378584 .617031$

H $\quad 35.627311134 .16184384 .769226$

N $\quad 37.689527134 .53518585 .241178$

$\mathrm{H} \quad 37.604536135 .27530885 .924023$

C $\quad 38.868240133 .96181784 .818210$

$\mathrm{H} \quad 39.825890134 .29362285 .171941$

$\mathrm{H} \quad 37.423974130 .30039781 .146894$

H $\quad 37.798218129 .95834382 .729570$

C $\quad 37.510288133 .49232377 .263855$

H $\quad 37.532142132 .42110277 .066360$

$\mathrm{H} \quad 37.155911133 .96334176 .340434$

C $\quad 36.516533133 .78937478 .395336$

$\mathrm{H} \quad 35.520513133 .53739378 .023754$

$\mathrm{H} \quad 36.463345134 .83831178 .691366$

C $\quad 36.688535132 .94804779 .648950$ 
O $\quad 36.691736131 .71466479 .561959$

O $\quad 36.784151133 .58591380 .777698$

C $\quad 34.425736130 .10553286 .585459$

H $\quad 35.284428130 .29708285 .932652$

H $\quad 34.799912130 .20881887 .608538$

C $\quad 33.423686131 .21988986 .382757$

N $\quad 33.319610131 .93536985 .190689$

C $\quad 32.517162132 .98276485 .417473$

$\mathrm{H} \quad 32.228201133 .74487384 .713454$

N $\quad 32.109730132 .98731986 .683461$

H $\quad 31.570254133 .77108087 .085192$

C $\quad 32.666121131 .89066987 .309015$

H $\quad 32.515172131 .69373388 .352694$

C $\quad 33.259820128 .48256379 .313789$

H $\quad 32.809972128 .80491178 .374056$

H $\quad 34.177620127 .93870779 .081280$

C $\quad 33.591007129 .67247680 .150824$

C $\quad 34.455128129 .86775381 .183698$

H $\quad 35.139733129 .17369181 .636209$

N $\quad 32.991095130 .90412279 .945525$

$\mathrm{H} \quad 32.292046131 .07521479 .227986$

C $\quad 33.474547131 .79362480 .812819$

$\mathrm{H} \quad 33.213036132 .83722180 .825301$

N $\quad 34.381783131 .19880481 .609865$

C $\quad 33.142890139 .81730181 .348554$

H $\quad 32.602302140 .47843380 .676288$

H $\quad 32.573273139 .77894282 .285748$

C $\quad 33.249923138 .42539380 .797002$

C $\quad 32.808102138 .11901279 .495576$

$\mathrm{H} \quad 32.377343138 .88846378 .873625$

C $\quad 32.889478136 .83932278 .988429$

H $\quad 32.528822136 .63255177 .987862$

C $\quad 33.755231137 .36117881 .582826$ 
$\begin{array}{ll}\mathrm{H} & 34.062685137 .56726282 .601382 \\ \mathrm{C} & 33.839284136 .07448881 .091514 \\ \mathrm{H} & 34.222488135 .26079981 .695316 \\ \mathrm{C} & 33.387739135 .81073379 .789986 \\ \mathrm{O} & 33.436491134 .51791079 .344457 \\ \mathrm{H} & 33.089973134 .46708778 .442600 \\ \mathrm{Cu} & 36.192463132 .15166582 .301590 \\ \mathrm{Cu} & 33.935868131 .52257283 .474245\end{array}$

The resting state of $\mathrm{Cu}^{\mathrm{I}} \mathrm{Cu}^{\mathrm{II}}$ at the BP86-D/TZVP level of theory

$\mathrm{E}=-4791.811090$ (a.u.)

N $\quad 37.651709130 .70147982 .040529$

C $\quad 38.917935131 .48847081 .891628$

H $\quad 38.624660132 .36070181 .285950$

C $\quad 39.432176131 .99624083 .234500$

H $\quad 40.399773132 .49603383 .094522$

H $\quad 39.649607131 .16023183 .920928$

C $\quad 38.507941132 .97683583 .922978$

$\mathrm{N} \quad 37.113798132 .97073183 .813434$

C $\quad 36.631631133 .91749284 .622674$

$\mathrm{H} \quad 35.582876134 .14776284 .774780$

$\mathrm{N} \quad 37.662655134 .54028385 .242729$

H $\quad 37.577474135 .29063585 .924869$

C $\quad 38.846705133 .96907584 .817455$

$\mathrm{H} \quad 39.812235134 .30672485 .167319$

$\mathrm{H} \quad 37.389194130 .33082481 .118991$

$\mathrm{H} \quad 37.785658129 .93332982 .703672$

C $\quad 37.505764133 .49000077 .277252$

$\mathrm{H} \quad 37.530057132 .41059877 .081194$

$\mathrm{H} \quad 37.143328133 .95445676 .344862$

C $\quad 36.513221133 .78280178 .415021$

$\mathrm{H} \quad 35.507564133 .53581878 .040358$

H $\quad 36.458117134 .83741478 .722014$ 
C $\quad 36.678732132 .92779079 .666842$

O $\quad 36.670446131 .68394079 .566915$

O $\quad 36.780448133 .56673880 .804713$

C $\quad 34.436893130 .10308486 .570904$

$\mathrm{H} \quad 35.299795130 .29175385 .910210$

$\mathrm{H} \quad 34.817880130 .21606987 .598691$

C $\quad 33.438259131 .22000486 .360093$

N $\quad 33.342286131 .93252585 .162993$

C $\quad 32.534393132 .99327785 .378577$

H $\quad 32.252824133 .75927284 .664791$

N $\quad 32.115738133 .00100486 .649987$

H $\quad 31.568513133 .79177187 .048435$

C $\quad 32.667653131 .90382487 .285323$

H $\quad 32.500818131 .70679188 .334864$

C $\quad 33.259170128 .49168679 .327455$

$\mathrm{H} \quad 32.804768128 .82266278 .384808$

H $\quad 34.181654127 .94614279 .084547$

C $\quad 33.600634129 .67666780 .169473$

C $\quad 34.480221129 .86740081 .203567$

$\mathrm{H} \quad 35.166716129 .16759881 .661705$

N $\quad 33.006978130 .91654279 .965384$

H $\quad 32.297584131 .08629379 .247897$

C $\quad 33.507261131 .81454980 .830029$

H $\quad 33.269456132 .87164880 .832215$

$\mathrm{N} \quad 34.424385131 .20582281 .623943$

C $\quad 33.143089139 .81621981 .347919$

H $\quad 32.594909140 .48300980 .676011$

$\mathrm{H} \quad 32.573719139 .77609982 .294814$

C $\quad 33.245547138 .42363180 .797734$

C $\quad 32.802441138 .11725879 .489979$

$\mathrm{H} \quad 32.363058138 .89039378 .865591$

C $\quad 32.896015136 .83515078 .975070$

H $\quad 32.530769136 .62868377 .967625$ 
C $\quad 33.757379137 .35278281 .585428$

H $\quad 34.072109137 .56051082 .609713$

C $\quad 33.853133136 .06294781 .086954$

H $\quad 34.253879135 .24355581 .686670$

C $\quad 33.394868135 .80076179 .781077$

O $\quad 33.427337134 .50006679 .340313$

H $\quad 33.084296134 .46074978 .426477$

$\mathrm{Cu} \quad 36.161846132 .12382282 .341588$

$\mathrm{Cu} \quad 34.014093131 .54953083 .474210$

The resting state of $\mathrm{Cu}^{\mathrm{I}} \mathrm{Cu}^{\mathrm{II}}$ at the B97-D/TZVP level of theory

$\mathrm{E}=-4791.600450$ (a.u.)

N $\quad 37.677982130 .68346382 .049781$

C $\quad 38.938994131 .48318281 .903741$

$\mathrm{H} \quad 38.636441132 .35119281 .307080$

C $\quad 39.450927131 .98886783 .250153$

$\mathrm{H} \quad 40.413544132 .48840283 .104421$

H $\quad 39.662372131 .15322583 .933543$

C $\quad 38.529960132 .97492883 .941792$

N $\quad 37.134341132 .96788483 .847588$

C $\quad 36.666109133 .92192384 .652732$

$\mathrm{H} \quad 35.624971134 .16236184 .817576$

N $\quad 37.702460134 .55041585 .255647$

H $\quad 37.626433135 .30169885 .931047$

C $\quad 38.880245133 .97285284 .821693$

$\mathrm{H} \quad 39.845204134 .31057185 .160339$

$\mathrm{H} \quad 37.426458130 .30022981 .137899$

H $\quad 37.812806129 .92938582 .720439$

C $\quad 37.522463133 .49895177 .229482$

$\mathrm{H} \quad 37.547848132 .42363577 .031766$

H $\quad 37.169242133 .97148376 .300827$

C $\quad 36.522706133 .78247378 .366328$

$\mathrm{H} \quad 35.525706133 .51815677 .990740$ 
$\mathrm{H} \quad 36.456874134 .83578078 .663884$

C $\quad 36.717993132 .93309379 .624918$

O $\quad 36.723901131 .69296479 .523407$

O 36.830204133 .57712580 .753990

C $\quad 34.421770130 .10116986 .597138$

H $\quad 35.274987130 .30169085 .931887$

H $\quad 34.804218130 .20770787 .622028$

C $\quad 33.414179131 .21579886 .399066$

$\mathrm{N} \quad 33.310166131 .92625985 .204019$

C $\quad 32.506642132 .98489885 .424761$

H $\quad 32.220974133 .74741684 .713523$

$\mathrm{N} \quad 32.097160132 .99674586 .698191$

$\mathrm{H} \quad 31.559965133 .78690787 .096714$

C $\quad 32.652748131 .90018187 .330626$

H $\quad 32.494179131 .70853688 .378094$

C $\quad 33.252345128 .46689279 .303906$

H $\quad 32.794322128 .79668678 .365744$

H $\quad 34.170828127 .91929179 .060977$

C $\quad 33.592317129 .65366580 .147906$

C $\quad 34.460180129 .84212681 .189503$

H $\quad 35.149391129 .14608181 .641376$

N $\quad 32.989412130 .88903779 .950719$

$\mathrm{H} \quad 32.289911131 .06170279 .231627$

C $\quad 33.473767131 .77822380 .831628$

$\mathrm{H} \quad 33.213525132 .82445580 .853385$

$\mathrm{N} \quad 34.385283131 .17331581 .627321$

C $\quad 33.134265139 .83205081 .349641$

H $\quad 32.589177140 .50074280 .681827$

$\mathrm{H} \quad 32.572460139 .78856482 .297493$

C $\quad 33.232632138 .43946680 .795732$

C $\quad 32.793951138 .13790979 .484140$

$\mathrm{H} \quad 32.361001138 .90983378 .860134$

C $\quad 32.883131136 .85713278 .968932$ 
$\begin{array}{ll}\mathrm{H} & 32.526364136 .64999877 .961996 \\ \mathrm{C} & 33.739066137 .36669081 .586353 \\ \mathrm{H} & 34.052587137 .57356382 .608101 \\ \mathrm{C} & 33.825009136 .07746981 .089832 \\ \mathrm{H} & 34.213867135 .25877781 .691723 \\ \mathrm{C} & 33.370746135 .81865279 .779360 \\ \mathrm{O} & 33.402154134 .52182679 .339861 \\ \mathrm{H} & 33.087009134 .47815878 .422484 \\ \mathrm{Cu} & 36.182999132 .11117682 .344803 \\ \mathrm{Cu} & 33.975973131 .51876683 .500846\end{array}$

The resting state of $\mathrm{Cu}^{\mathrm{I}} \mathrm{Cu}^{\mathrm{II}}$ at the M06-D/TZVP level of theory

$\mathrm{E}=-4789.932221$ (a.u.)

$\mathrm{N} \quad 37.622711130 .72066081 .960821$

C $\quad 38.880784131 .49482081 .864464$

$\mathrm{H} \quad 38.616672132 .38268181 .277460$

C $\quad 39.365600131 .95416683 .221211$

$\mathrm{H} \quad 40.361110132 .39099983 .130535$

$\mathrm{H} \quad 39.499309131 .09863883 .894727$

C $\quad 38.481263132 .97091683 .885531$

$\mathrm{N} \quad 37.100143133 .01067683 .756908$

C $\quad 36.652136133 .96725684 .546619$

$\mathrm{H} \quad 35.618448134 .24942584 .684984$

$\mathrm{N} \quad 37.681283134 .55581985 .174805$

$\mathrm{H} \quad 37.611678135 .30266785 .850341$

C $\quad 38.840910133 .94540984 .769778$

$\mathrm{H} \quad 39.805911134 .24621285 .133281$

$\mathrm{H} \quad 37.352740130 .42097481 .025380$

$\mathrm{H} \quad 37.753971129 .91523782 .563621$

C $\quad 37.501065133 .51729377 .252399$

H $\quad 37.518130132 .44440377 .062597$

$\mathrm{H} \quad 37.157767133 .97622776 .319141$

C $\quad 36.511999133 .82294078 .366611$ 
$\mathrm{H} \quad 35.515270133 .58153477 .982963$

$\mathrm{H} \quad 36.463102134 .87259278 .669030$

C $\quad 36.660428132 .97705079 .612582$

O $\quad 36.668550131 .75126679 .501719$

O $\quad 36.733050133 .60291880 .735689$

C $\quad 34.434348130 .10886786 .559171$

H $\quad 35.301416130 .29193385 .913912$

H $\quad 34.804558130 .21946587 .583849$

C $\quad 33.441419131 .21303586 .349537$

$\mathrm{N} \quad 33.314846131 .90409985 .152451$

C $\quad 32.518253132 .94862085 .378329$

H $\quad 32.205012133 .69761784 .666831$

$\mathrm{N} \quad 32.133021132 .97574686 .646381$

H $\quad 31.598391133 .76524387 .041934$

C $\quad 32.699761131 .89440587 .274456$

H $\quad 32.564268131 .70703688 .323893$

C $\quad 33.268891128 .48443479 .315007$

H $\quad 32.818773128 .80436478 .374415$

$\mathrm{H} \quad 34.179291127 .93159179 .077105$

C $\quad 33.607048129 .66663380 .141395$

C $\quad 34.459545129 .85458181 .180721$

H $\quad 35.133248129 .14917481 .638609$

$\mathrm{N} \quad 33.030607130 .89965279 .921048$

$\mathrm{H} \quad 32.338377131 .07428379 .199752$

C $\quad 33.516899131 .78101180 .791086$

H $\quad 33.262559132 .82937880 .804275$

$\mathrm{N} \quad 34.401040131 .18199581 .596416$

C $\quad 33.150159139 .81121681 .332704$

$\mathrm{H} \quad 32.625072140 .47276680 .648563$

$\mathrm{H} \quad 32.566502139 .78609382 .262401$

C $\quad 33.246680138 .42777180 .795751$

C $\quad 32.798994138 .12225879 .495800$

H $\quad 32.377106138 .89816078 .874902$ 
C $\quad 32.871361136 .85136478 .993385$

H $\quad 32.511106136 .64089377 .992655$

C $\quad 33.739391137 .35980481 .585031$

H $\quad 34.051799137 .56993782 .602582$

C $\quad 33.811862136 .08055881 .098317$

H $\quad 34.186999135 .25799881 .700680$

C $\quad 33.356538135 .81835079 .796666$

O $\quad 33.389654134 .54623279 .355990$

H $\quad 33.053812134 .49901878 .450842$

$\mathrm{Cu} \quad 36.150106132 .15272082 .269003$

$\mathrm{Cu} \quad 33.968274131 .51594283 .453039$

The $\mu-\eta^{2}: \eta^{2}$-peroxo- $\mathrm{Cu}_{2}^{\mathrm{II}}$ state (triplet)

$\mathrm{E}=-4940.654122$ (a.u.)

O $\quad 37.519047129 .83410584 .198190$

O $\quad 36.812720129 .69276082 .992364$

$\mathrm{N} \quad 39.352574129 .76786480 .835339$

C $\quad 40.759711130 .14742780 .508096$

H $\quad 40.885816131 .16836780 .871328$

C $\quad 41.780334129 .23752181 .187022$

H $\quad 41.561191128 .18915680 .934766$

H $\quad 42.787021129 .45559280 .816440$

C $\quad 41.812636129 .39185982 .671732$

$\mathrm{N} \quad 40.679408129 .54185583 .464238$

C $\quad 41.091618129 .64108584 .719516$

$\mathrm{H} \quad 40.456733129 .81039085 .572192$

N $\quad 42.429763129 .55053984 .768642$

H $\quad 43.012876129 .61931585 .591176$

C $\quad 42.906788129 .41114283 .484017$

$\mathrm{H} \quad 43.956255129 .38194183 .244520$

H $\quad 38.701492130 .45676180 .361352$

$\mathrm{H} \quad 39.147224128 .85680980 .428998$

C $\quad 37.046434133 .91235777 .769444$ 


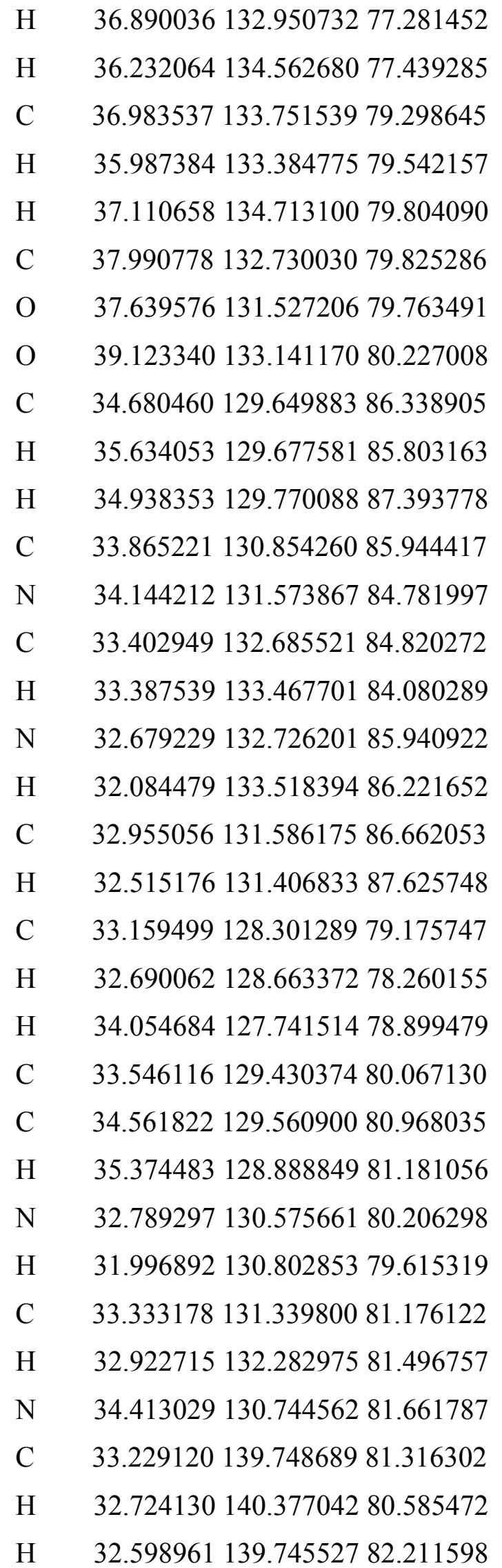


C $\quad 33.369691138 .32555280 .811703$

C $\quad 32.859909137 .94261579 .568474$

$\mathrm{H} \quad 32.370297138 .67145778 .934926$

C $\quad 32.928991136 .62750479 .117394$

H $\quad 32.509674136 .36193778 .156329$

C $\quad 33.947464137 .32353581 .601797$

H $\quad 34.322010137 .57207982 .588727$

C $\quad 34.024538136 .00458281 .165369$

$\mathrm{H} \quad 34.451106135 .24165081 .812633$

C $\quad 33.502787135 .65486879 .922221$

O $\quad 33.501797134 .34024779 .467588$

$\mathrm{H} \quad 34.132179133 .83648679 .992722$

$\mathrm{Cu} \quad 38.847940129 .82839282 .758032$

$\mathrm{Cu} \quad 35.367640130 .90085683 .414887$

The $\mu-\eta^{2}: \eta^{2}$-peroxo- $-\mathrm{Cu}_{2}{ }_{2}$ state (open-shell singlet)

$\mathrm{E}=-4940.657214$ (a.u.)

O $\quad 37.276832130 .24412684 .032235$

O $\quad 36.948940130 .10695282 .618045$

N $\quad 39.370148129 .80512880 .857483$

C $\quad 40.781152130 .14923880 .532062$

$\mathrm{H} \quad 40.934650131 .16928480 .887652$

C $\quad 41.763498129 .21135381 .225930$

H $\quad 41.516080128 .17020280 .968369$

$\mathrm{H} \quad 42.781847129 .39946780 .871844$

C $\quad 41.775810129 .36936682 .712318$

$\mathrm{N} \quad 40.643049129 .59103183 .494332$

C $\quad 41.051895129 .68468484 .752378$

$\mathrm{H} \quad 40.424524129 .91488885 .596087$

$\mathrm{N} \quad 42.382097129 .51964384 .814384$

$\mathrm{H} \quad 42.969775129 .58687285 .634746$

C $\quad 42.860605129 .33923583 .536088$

H $\quad 43.909988129 .25430783 .309902$ 
H $\quad 38.723279130 .47351980 .343011$

H $\quad 39.159947128 .87272980 .506044$

C $\quad 37.068976133 .90624877 .767886$

H $\quad 36.909708132 .94634677 .278177$

H $\quad 36.254979134 .56030977 .444098$

C $\quad 37.015571133 .73557879 .296406$

H $\quad 36.018782133 .37360179 .547696$

$\mathrm{H} \quad 37.153490134 .69141979 .810003$

C $\quad 38.026113132 .70323779 .794340$

O $\quad 37.708505131 .50065579 .633812$

O $\quad 39.134705133 .10844080 .264860$

C $\quad 34.718854129 .63163486 .320351$

$\mathrm{H} \quad 35.659956129 .65192185 .764533$

H $\quad 34.990807129 .75965587 .371176$

C $\quad 33.901405130 .83390985 .926992$

$\mathrm{N} \quad 34.170212131 .55054684 .762696$

C $\quad 33.422025132 .65627384 .796883$

H $\quad 33.403879133 .43470684 .053283$

$\mathrm{N} \quad 32.699571132 .69657185 .919271$

H $\quad 32.101262133 .48677286 .198777$

C $\quad 32.985495131 .56148186 .643587$

H $\quad 32.548717131 .38087287 .608822$

C $\quad 33.121213128 .32185679 .221936$

$\mathrm{H} \quad 32.664051128 .68108478 .299850$

H $\quad 34.037438127 .78696078 .963928$

C $\quad 33.461413129 .45437280 .127421$

C $\quad 34.471164129 .60921281 .028970$

$\mathrm{H} \quad 35.309118128 .96818181 .235325$

$\mathrm{N} \quad 32.673343130 .57809880 .271810$

$\mathrm{H} \quad 31.882315130 .78928679 .674430$

C $\quad 33.195654131 .35539581 .244346$

H $\quad 32.764971132 .28902581 .565526$

N $\quad 34.289335130 .78685081 .728260$ 
C $\quad 33.227043139 .74498581 .314162$

H $\quad 32.720909140 .37487480 .585370$

$\mathrm{H} \quad 32.597697139 .73908682 .210000$

C $\quad 33.365817138 .32251080 .805119$

C $\quad 32.862880137 .94455979 .557192$

H $\quad 32.375625138 .67554378 .924153$

C $\quad 32.934400136 .63138179 .100468$

H $\quad 32.518399136 .37004378 .136644$

C $\quad 33.934153137 .31544481 .595540$

$\mathrm{H} \quad 34.299837137 .55818982 .587160$

C $\quad 34.014269135 .99839681 .153198$

$\mathrm{H} \quad 34.436540135 .23207981 .799556$

C $\quad 33.503551135 .65440479 .904031$

O $\quad 33.511637134 .34383079 .439873$

H 34.095491133 .82370480 .001875

$\mathrm{Cu} \quad 38.852070129 .96895482 .773057$

$\mathrm{Cu} \quad 35.412745130 .96796083 .372069$ 